\title{
Psychological Features of Smokers
}

\author{
Askin Gülsen MD and Bülent Uygur MD
}

\begin{abstract}
BACKGROUND: Smoking tobacco is believed to be common among patients with psychiatric disorders like anxiety, depression, schizophrenia, and affective disorders. The aim of this study was to investigate the psychological symptoms of people without known psychiatric diseases who have freely decided to quit smoking. METHODS: A cross-sectional study was designed, and 124 healthy activesmokers (66 female) and 127 healthy non-smokers (61 female) were recruited. The symptom check list and Fagerström Test for Nicotine Dependence (FTND) were used to assess psychological symptoms and nicotine addiction level. Smokers were divided into 3 groups: light smokers (0-9 cigarettes/d), moderate smokers (10-19 cigarettes/d), and heavy smokers (> 20 cigarettes/d). RESULTS: When subjects were compared by daily levels of cigarette consumption, somatic findings were more prominent in the mild smoker group $(1.61 \pm 0.72$ vs $0.77 \pm 0.56, P<.001)$, while depression and hostility were similar across all groups. When subjects were compared by nicotine addiction ratio, the general symptom index of the group with high-grade nicotine addiction was found to be significantly higher than that of the control group $(1.34 \pm 0.72$ vs $0.79 \pm 0.49, P<.001)$. In addition, the high-grade nicotine addiction group had higher scores than the other groups for somatization, anxiety, depression, paranoid symptoms, hostility, and other symptoms. CONCLUSION: This study examined the association between psychological symptoms and smokers' daily cigarette consumption and nicotine addiction levels. Psychiatric symptoms (ie, somatization, anxiety, depression, hostility, and paranoia) were observed more frequently in people with high-grade nicotine addiction (FTND score 7-10 points). Key words: psychiatric disorders; nicotine addiction; symptom check list-90; fagerström; smoking; tobacco; psychological features. [Respir Care 2018;63(12):1492-1497. () 2018 Daedalus Enterprises]
\end{abstract}

\section{Introduction}

Cigarette smoking has become an increasingly severe health problem in recent years, such that deaths are expected to increase 10 -fold to $>8$ million people by 2030 in worldwide. ${ }^{1,2}$ Nicotine addiction is very common among patients with psychiatric disorders, affecting $35-80 \%$ of them. ${ }^{3}$ As a result, these patients have been found to die $20-$ $30 \mathrm{y}$ earlier than the general population. ${ }^{4}$ Smoking is

Dr Gülsen is affiliated with the Division of Clinical and Molecular A1lergology, Research Center Borstel, Leibniz Lung Center, Borstel, Germany. Dr Uygur is affiliated with the Department of Psychiatry, Private Medifema Hospital, Izmir, Turkey.

The authors have disclosed no conflicts of interest.

Correspondence: Askin Gülsen MD, Research Center Borstel, Leibniz Lung Center, Parkallee 35, 23845 Borstel, Germany. E-mail: askingulsen@ hotmail.com.

DOI: $10.4187 /$ respcare. 06287 associated with many diseases. It leads to negative health outcomes and remains a major social health problem.

Among patients diagnosed with major psychiatric disorders, schizophrenia patients have the highest smoking rates at $40-90 \%$. These patients are highly resistant to smoking cessation treatments. ${ }^{5,6} \mathrm{~A}$ high proportion of patients with psychotic, panic, and obsessive-compulsive disorders and depression are smokers, and smoking cessation has been shown to worsen their disease symptoms. ${ }^{7}$ The smoking rates associated with psychiatric disorders as determined by various studies are shown in Table 1 . Nicotine addiction is now included in the substance use disorders section of the Diagnostic and Statistical Manual (DSM-V), ${ }^{8}$ the standard classification guide for psychiatric disorders.

Today, smokers often visit specialized clinics or chest disease clinics when they suffer from medical conditions or have recently stopped smoking. These out-patient clinics not only treat patients but also evaluate their potential to quit smoking. A number of studies have previously reported that smoking and nicotine addiction are associated with depression and anxiety; smoking cessation has 
Table 1. Psychiatric Disorders and Rarity of Smoking

\begin{tabular}{lcc}
\hline \hline Psychiatric Disorders & Smoking Rate, $\%$ & References \\
\hline Obsessive-compulsive & $13-37$ & 25,26 \\
Anxiety & $17-47$ & $16,23,27$ \\
Somatoform & 48 & 18 \\
Depression & $18-60$ & 17 \\
Bipolar disorder & $52-70$ & $16,18,23$ \\
Psychoticism & $57-66$ & 28,29 \\
Schizophrenia & $40-90$ & $5,6,16$ \\
\hline
\end{tabular}

also been found to reduce depression. ${ }^{9}$ For this reason, patients are often evaluated in out-patient clinics only for depression and anxiety. The Fagerström Test for Nicotine Dependence (FTND) is the most often used test to determine nicotine addiction level. ${ }^{10}$

However, none of these surveys were able to adequately evaluate patients' other psychological symptoms. Given that smoking and psychiatric disorders are interrelated, it is clear that psychiatric findings relating to patients should be evaluated more objectively and patients needing psychiatric support should be detected earlier. A review of the literature reveals that cigarette use is insufficiently evaluated or documented in persons with psychiatric diseases such as somatoform conditions, personality disorders, or panic disorder.

The aim of this study was to investigate the psychological symptoms of people without known psychiatric diseases who have freely decided to quit smoking. In particular, this study examines whether the number of cigarettes consumed per day and the degree of nicotine addiction are related to psychological symptoms.

\section{Methods}

\section{Study Population}

This was an observational cross-sectional study that was conducted from May to August 2015 in Turkey; data were gathered using a questionnaire filled out by subjects who attended a psychiatry polyclinic to quit smoking. The study included 2 groups: Group 1 was made up of 124 subjects and Group 2 consisted of 127 healthy volunteers who had never smoked. The healthy study population was composed of healthy volunteers, patient relatives, and hospital staff. The study was conducted according to good clinical practice and the Declaration of Helsinki. Protocol approval was obtained from an independent local ethics committee. The study patients were informed about the research, and consent forms were obtained.

\section{Inclusion and Exclusion Criteria}

Subjects who met inclusion criteria included persons visiting out-patient clinics to quit smoking who were 18

\section{QUICK LOOK}

\section{Current knowledge}

Cigarette smoking has become an increasingly severe health problem and deaths are expected to increase 10fold by 2030 worldwide. Among patients with psychiatric disorders, nicotine addiction is very common, affecting $35-80 \%$ of such individuals. Today, smokers often visit specialized polyclinics or chest disease polyclinics when they suffer from medical conditions or have recently stopped smoking. As these out-patient clinics treat patients, they also evaluate their potential to quit smoking.

\section{What this paper contributes to our knowledge}

Psychiatric symptoms were observed more frequently in subjects with severe nicotine addiction. The psychological symptom questionnaire may be important in identifying patients who need psychiatric counseling.

65 y old. Subjects who had known psychiatric disorders, were receiving psychiatric treatment, had alcohol or substance addiction, were suffering from chronic lung disease (eg, asthma, COPD, interstitial lung disease, sarcoidosis, bronchiectasis), or were suffering from a comorbidity (eg, diabetes mellitus, coronary artery disease, hearth failure, hypertension) were excluded from the study.

\section{Data Collection}

Each subject was asked to fill out a psychological symptom questionnaire known as the Symptom Check List 90revised (the SCL-90-R) and the FTND using the face-toface interview technique. ${ }^{10,11}$ The SCL-90-R, developed by Derogatis ${ }^{11}$ is a psychiatric screening survey containing 90 items and 9 subheadings: somatic findings, depression, anxiety, obsessiveness, interpersonal sensitivity, anger, paranoia symptoms, psychotic symptoms, additional indices, and the general symptom index. Each item on the test is rated on a 5-point Likert scale with the following options: not at all/a little/moderately/quite a bit/very much. Subject responses were scored on a scale of $0-4$. A score of $\geq 1$ indicates possible psychopathology. The validity and reliability of the Turkish version of the form was checked and confirmed by Dağ. ${ }^{12}$ The other questionnaire, the FTND, is the most valid and commonly used scale for measuring nicotine addiction. ${ }^{10}$ The validity and reliability of the Turkish version of the scale was checked and confirmed by Uysal et al. ${ }^{13}$ The questionnaire consists of 6 questions, each scored separately. The highest possible score is 10 points. In our study, nicotine addictions were categorized as low-grade ( $0-3$ points), intermediategrade (4-6 points), and high-grade (7-10 points). Subjects 


\section{Psychological Features of SMOKers}

were divided into 3 groups according to their daily cigarette consumption as mild smokers (0-9 cigarettes/d), moderate smokers (10-19 cigarettes/d), and heavy smokers $(\geq 20$ cigarettes/d).

\section{Statistical Analysis}

Statistical analysis was carried out using SPSS version 18 (SPSS, Chicago, Illinois). A chi-square test was used to compare the demographic data, a Student $t$ test was applied to define the parameters of normal distribution groups, and the Mann-Whitney $\mathrm{U}$ test was used for non-normal distribution parameters. $P<.05$ was considered statistically significant in the Student $t$ test, and $P<.01$ in the Mann-Whitney U test.

\section{Results}

The mean age of the 124 subjects ( 66 women and 58 men) in the treatment group was $42.2 \pm 12.3$ y (range 19-64 y) The control group consisted of 127 subjects (61 women and 66 men) with a mean age of $41.5 \pm 12.9$ y (range $18-67 \mathrm{y}$ ). The average age at which people had started smoking was $18.4 \pm 4.1 \mathrm{y}$ (range $9-40 \mathrm{y}$ ). The average level of cigarette consumption was 25.6 pack-years.

By average daily levels of cigarette consumption, 7 of 124 (5.6\%) of the subjects were mild smokers, 39 of 124 (31.5\%) were moderate smokers, and 78 of 124 (62.9\%) were heavy smokers. Analysis of the FTND responses found the following distribution of subjects by level of nicotine addiction: 41 of $124(33.1 \%)$ had a low-grade addiction, 56 of 124 $(45.1 \%)$ had an intermediate-grade addiction, and 27 of 124 $(21.8 \%)$ a high-grade addiction (Table 2).

Across all groups, the overall index of symptoms was found to be significantly more prominent than in the control group $(1.02 \pm 0.63$ and $0.79 \pm 0.49, P<.001)$. In addition, the smokers' symptom scores for psychiatric indications such as somatization, depression, and hostility were significantly higher (Table 3 ).

When subjects were compared by daily level of cigarette consumption, somatic findings were more prominent in the mild smoker group $(1.61 \pm 0.72$ vs $0.77 \pm 0.56, P<.001)$, whereas depression and hostility findings were similar across all groups (Fig. 1). Although obsessive symptoms had scores $>1$ in all groups, including the control group, there was no statistical difference between the groups.

When subjects were compared by nicotine addiction ratio, the general symptom index of the group with highgrade nicotine addiction was found to be significantly higher than that of the control group $(1.34 \pm 0.72$ vs $0.79 \pm 0.49$, $P<.001$ ) (Fig. 2). In addition, the high-grade nicotine addiction group had higher scores than the other groups for somatization, anxiety, depression, paranoid symptoms, hostility, and other symptoms (Table 4).
Table 2. Demographic Features of Study Population

\begin{tabular}{|c|c|c|c|}
\hline & $\begin{array}{c}\text { Group 1 } \\
\text { (Smokers) } \\
n=124\end{array}$ & $\begin{array}{c}\text { Group 2 } \\
\text { (Control) } \\
n=127\end{array}$ & $P$ \\
\hline \multicolumn{4}{|l|}{ Sex, $n(\%)$} \\
\hline Female & $66(46.8)$ & $61(48)$ & \\
\hline Male & $58(53.2)$ & $66(52)$ & \\
\hline Age, y & $42.2 \pm 12.3$ & $41.5 \pm 12.9$ & \\
\hline Age at start smoking, y & $18.4 \pm 4.1$ & & \\
\hline Daily number of cigarettes & $25.6 \pm 22.3$ & & \\
\hline Light smoker (0-9) & $7(5.6)$ & & \\
\hline Medium smoker (10-19) & $39(31.5)$ & & \\
\hline Heavy smoker $(>20)$ & $78(62.9)$ & & \\
\hline \multicolumn{4}{|l|}{ FTND, points } \\
\hline Minimally dependent (0-3) & $41(33.1)$ & & \\
\hline Moderately dependent (4-6) & $56(45.1)$ & & \\
\hline Highly dependent (7-10) & $27(21.8)$ & & \\
\hline \multicolumn{4}{|l|}{ SCL-90-R } \\
\hline Somatization & $1.34 \pm 0.82$ & $0.77 \pm 0.56$ & $<.001$ \\
\hline Anxiety & $0.93 \pm 0.71$ & $0.70 \pm 0.55$ & .006 \\
\hline Obsessive-compulsive & $1.25 \pm 0.75$ & $1.21 \pm 0.63$ & .64 \\
\hline Depression & $1.23 \pm 0.80$ & $0.87 \pm 0.64$ & $<.001$ \\
\hline Interpersonal sensitivity & $0.95 \pm 0.71$ & $1.04 \pm 0.76$ & .33 \\
\hline Psychoticism & $0.64 \pm 0.58$ & $0.45 \pm 0.45$ & .004 \\
\hline Paranoid ideation & $0.97 \pm 0.78$ & $0.81 \pm 0.61$ & .09 \\
\hline Hostility & $1.17 \pm 0.96$ & $0.70 \pm 0.64$ & $<.001$ \\
\hline Phobic anxiety & $0.55 \pm 0.61$ & $0.42 \pm 0.50$ & .07 \\
\hline Additional scales & $1.12 \pm 0.73$ & $0.79 \pm 0.60$ & $<.001$ \\
\hline Global severity index & $1.02 \pm 0.63$ & $0.79 \pm 0.49$ & .001 \\
\hline \multicolumn{4}{|c|}{$\begin{array}{l}\text { Data are presented as mean } \pm \text { SD or } n(\%) \text {, possible psychopathology }>1 . P<.05 \text { was } \\
\text { statistically significant. } \\
\text { FTND }=\text { Fagerström Test for Nicotine Dependence } \\
\text { SCL-90-R = Symptom Check List } 90 \text { revised }\end{array}$} \\
\hline
\end{tabular}

\section{Discussion}

This study demonstrates that daily cigarette smoking is associated with somatization, depression, and hostility. Somatic symptoms are especially prominent among mild and moderate smokers. Interestingly, as daily cigarette smoking increases, psychiatric symptoms do not show a proportional or linear increase. This result suggests that people may be trying to cure themselves, suppress negative emotions, or relieve sadness or stress by smoking. There is evidence in the literature that nicotine use affects neurochemical systems and improves mood. ${ }^{14}$ People who stop smoking frequently experience withdrawal symptoms such as anger, difficulty concentrating, nervousness, agitation, and anxiety during the first 4-6 weeks after quitting. ${ }^{8,15}$ These symptoms have been found to last for about $30 \mathrm{~d}$ during the period of cessation, and compulsive symptoms may last for months or even years. ${ }^{7}$ However, other studies have shown that people with high levels of psychological stress are more likely to smoke. ${ }^{16}$ Research has also found 
Table 3. Comparison of Psychological Symptoms With Daily Cigarette Consumption

\begin{tabular}{|c|c|c|c|c|c|}
\hline SCL-90-R & $\begin{array}{c}\text { Light Smoker } \\
(0-9 \text { cigarettes/d) }\end{array}$ & $\begin{array}{l}\text { Medium Smoker } \\
\text { (10-19 cigarettes/d) }\end{array}$ & $\begin{array}{c}\text { Heavy Smoker } \\
(>20 / d \text { cigarettes } / d)\end{array}$ & $\begin{array}{l}\text { Control } \\
\text { Group }\end{array}$ & $P$ \\
\hline Somatization & $1.61 \pm 0.72^{\mathrm{a}}$ & $1.42 \pm 0.91^{\mathrm{a}}$ & $1.27 \pm 0.78^{\mathrm{a}}$ & $0.77 \pm 0.56$ & $<.001$ \\
\hline Anxiety & $0.80 \pm 0.55$ & $1.05 \pm 0.77$ & $0.90 \pm 0.69^{\mathrm{a}}$ & $0.70 \pm 0.55$ & .035 \\
\hline Obsessive-compulsive & $1.17 \pm 0.80$ & $1.22 \pm 0.74$ & $1.27 \pm 0.81^{\mathrm{a}}$ & $1.21 \pm 0.63$ & .54 \\
\hline Depression & $1.26 \pm 0.93^{\mathrm{a}}$ & $1.19 \pm 0.79^{\mathrm{a}}$ & $1.24 \pm 0.81^{\mathrm{a}}$ & $0.87 \pm 0.64$ & .001 \\
\hline Interpersonal sensitivity & $0.74 \pm 0.56$ & $0.83 \pm 0.64$ & $1.03 \pm 0.75^{\mathrm{a}}$ & $1.04 \pm 0.76$ & .91 \\
\hline Psychoticism & $0.64 \pm 0.38$ & $0.57 \pm 0.59$ & $0.68 \pm 0.59^{\mathrm{a}}$ & $0.45 \pm 0.45$ & .005 \\
\hline Paranoid ideation & $0.83 \pm 0.63$ & $0.91 \pm 0.83$ & $1.01 \pm 0.70^{\mathrm{a}}$ & $0.81 \pm 0.61$ & .065 \\
\hline Hostility & $1.40 \pm 1.19^{\mathrm{a}}$ & $1.04 \pm 0.98$ & $1.21 \pm 0.94^{\mathrm{a}}$ & $0.70 \pm 0.64$ & $<.001$ \\
\hline Phobic anxiety & $0.50 \pm 0.75$ & $0.53 \pm 0.54$ & $0.57 \pm 0.64^{\mathrm{a}}$ & $0.42 \pm 0.50$ & .09 \\
\hline Additional scales & $0.95 \pm 0.83$ & $1.14 \pm 0.73^{\mathrm{a}}$ & $1.12 \pm 0.72^{\mathrm{a}}$ & $0.79 \pm 0.60$ & .001 \\
\hline Global severity index & $1.02 \pm 0.61^{\mathrm{a}}$ & $1.01 \pm 0.63^{\mathrm{a}}$ & $1.05 \pm 0.63^{\mathrm{a}}$ & $0.79 \pm 0.49$ & .003 \\
\hline \multicolumn{6}{|c|}{$\begin{array}{l}\text { Data are presented as mean } \pm \mathrm{SD} \text {, possible psychopathology }>1 . P<.05 \text { was considered statistically significant in the Student } t \text { test and } P<.01 \text { in the Mann-Whitney } \mathrm{U} \text { test. } \\
\text { From Table 2: Light smokers, } n=7 \text {; medium smokers, } n=39 \text {; heavy smokers, } n=78 \text {; control group, } n=127 \text {. } \\
\text { a Comparison between group and control group. } \\
\text { SCL-90-R }=\text { Symptom Check List } 90 \text { revised }\end{array}$} \\
\hline
\end{tabular}

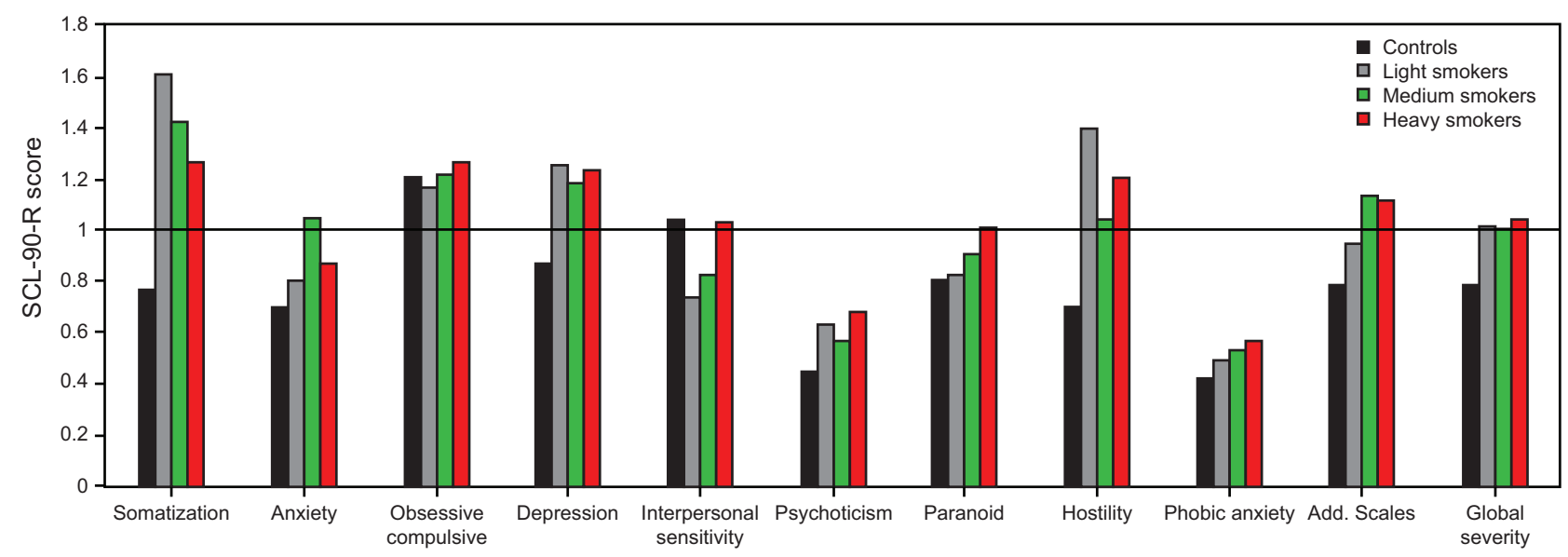

Fig. 1. Comparison of psychological symptoms with daily cigarette consumption. Controls $(n=127)$; light smokers, $0-9$ cigarettes/d $(n=$ 7); moderate smokers, $10-19$ cigarettes/d $(n=39)$; heavy smokers, $>20$ cigarettes/d $(n=78)$. Values $>1$ indicate possible psychopathology.

that when smokers with a history of major depression quit smoking, their depressive symptoms may increase in the first 12 months after quitting. ${ }^{17}$ In our study, symptoms of hostility were high among smokers. However, these symptoms did not increase in line with the daily amount smoked. More detailed studies are needed to investigate the links between anger control and smoking.

There are very few studies of smoking and somatoform disorders in the literature. In one study, the rate of smoking in a group of subjects with somatoform disorders was $48 \%$, and cigarette smoking was relegated to a secondary position due to the psychiatric disease-focused treatment management. ${ }^{18}$ The high level of somatic symptoms among smokers may reflect their strong expectation of illness.

The results of our study show that somatization, anxiety, depression, anger, and paranoia symptoms were more frequent among subjects with high-grade nicotine addiction (FTND score 7-10). These psychiatric symptoms increase with the subjects' level of nicotine addiction. It is known that patients with particularly severe levels of nicotine addiction have difficulty giving up smoking and struggle to find the motivation to quit. Today, special treatments are recommended for these patients in accordance with their degree of motivation. ${ }^{19,20}$ We believe that when treatment fails in this group of patients, a detailed evaluation of the psychiatric findings before and after the program, alongside treatment of anxiety, depression, and somatic and paranoid behavior, can be beneficial, as can anger control and psychiatric counseling. A study conducted by Becoña et $\mathrm{al}^{21}$ found that smoking rates were higher among people with paranoia or narcissistic, antisocial, or borderline personality disorders. 


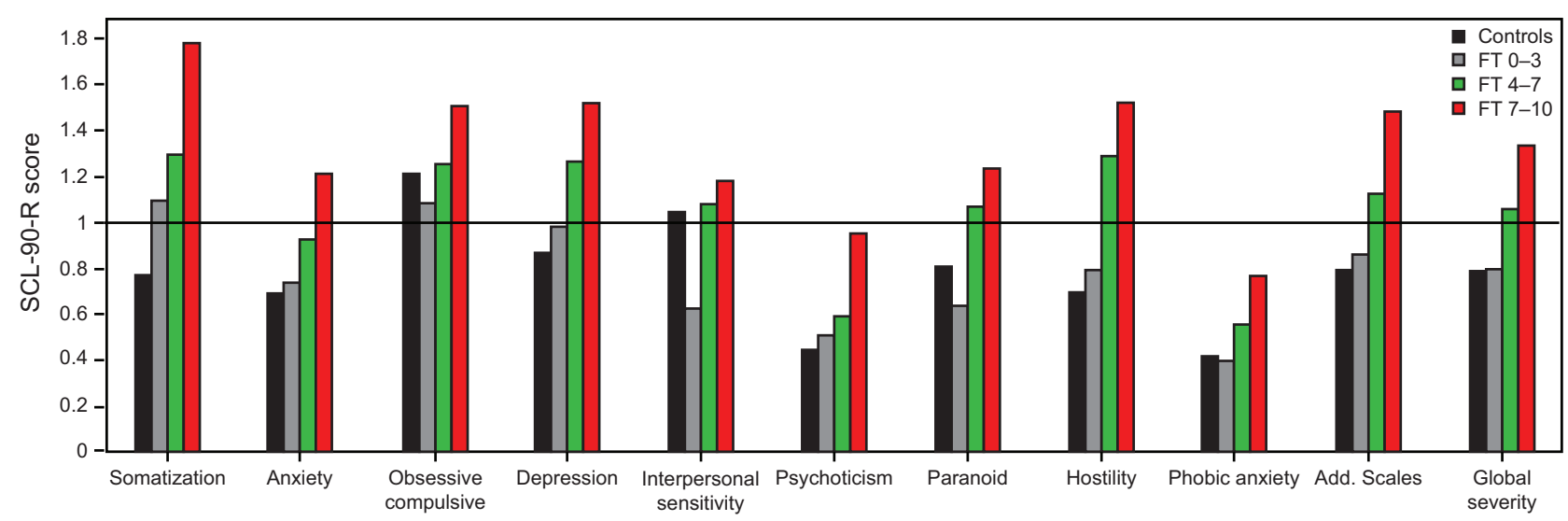

Fig. 2. Comparison of psychological symptoms with nicotine addiction level. Controls ( $n=127$ ); Fagerström test, $0-3$ ( $n=41)$; Fagerström test, 4-7 $(n=56)$; and Fagerström test, $7-10(n=27)$. Values $>1$ indicate possible psychopathology.

Table 4. Comparison of Psychological Symptoms With Nicotine Addiction Level

\begin{tabular}{|c|c|c|c|c|c|}
\hline SCL-90-R & FTND $(0-3)$ & FTND (4-6) & FTND (7-10) & Control Group & $P$ \\
\hline Somatization & $1.10 \pm 0.84$ & $1.30 \pm 0.74^{\mathrm{a}}$ & $1.78 \pm 0.79^{\mathrm{a}}$ & $0.77 \pm 0.56$ & $<.001$ \\
\hline Anxiety & $0.74 \pm 0.63$ & $0.93 \pm 0.65$ & $1.21 \pm 0.86^{\mathrm{a}}$ & $0.70 \pm 0.55$ & .007 \\
\hline Obsessive-compulsive & $1.08 \pm 0.74$ & $1.25 \pm 0.69$ & $1.51 \pm 0.83^{\mathrm{a}}$ & $1.21 \pm 0.63$ & .09 \\
\hline Depression & $0.98 \pm 0.70$ & $1.27 \pm 0.75^{\mathrm{a}}$ & $1.52 \pm 0.95^{\mathrm{a}}$ & $0.87 \pm 0.64$ & .002 \\
\hline Interpersonal sensitivity & $0.63 \pm 0.52$ & $1.08 \pm 0.68$ & $1.18 \pm 0.86^{\mathrm{a}}$ & $1.04 \pm 0.76$ & .47 \\
\hline Psychoticism & $0.51 \pm 0.49$ & $0.59 \pm 0.51$ & $0.95 \pm 0.73^{\mathrm{a}}$ & $0.45 \pm 0.45$ & .002 \\
\hline Paranoid ideation & $0.64 \pm 0.59$ & $1.07 \pm 0.72$ & $1.24 \pm 0.99^{\mathrm{a}}$ & $0.81 \pm 0.61$ & .041 \\
\hline Hostility & $0.79 \pm 0.82$ & $1.28 \pm 0.95^{\mathrm{a}}$ & $1.52 \pm 1.02^{\mathrm{a}}$ & $0.70 \pm 0.64$ & $<.001$ \\
\hline Phobic anxiety & $0.40 \pm 0.47$ & $0.56 \pm 0.54$ & $0.77 \pm 0.86^{\mathrm{a}}$ & $0.42 \pm 0.50$ & .053 \\
\hline Additional scales & $0.87 \pm 0.63$ & $1.12 \pm 0.70$ & $1.48 \pm 0.77^{\mathrm{a}}$ & $0.79 \pm 0.60$ & $<.001$ \\
\hline Global severity index & $0.80 \pm 0.54$ & $1.06 \pm 0.59$ & $1.34 \pm 0.72^{\mathrm{a}}$ & $0.79 \pm 0.49$ & .001 \\
\hline
\end{tabular}

Data are presented as mean $\pm \mathrm{SD}$, possible psychopathology $>1 . P<.05$ was considered statistically significant in the Student $t$ test and $P<.01$ in the Mann-Whitney $\mathrm{U}$ test.

From Table 2: FTND (0-3), $n=41$; FTND (4-6), $n=56$; FTND (7-10), $n=27$; control group, $n=127$.

${ }^{\text {a }}$ Comparison between group and control group.

FTND $=$ Fagerström Test for Nicotine Dependence

SCL-90-R = Symptom Check List 90 revised

The interrelatedness of smoking and some psychiatric disorders (eg, depressive and anxiety disorders) is well recognized. ${ }^{22}$ Studies have shown that smoking and anxiety are closely related, with a joint prevalence of $14-47 \% .16,23,24$ The relationship between smoking and other psychiatric disorders (personality, somatoform, panic, and bipolar disorders) has received little attention, however, and it is rarely explored despite the fact that the overall smoking rate in the psychiatric patient population is $41-52 \%$, significantly higher than in the normal population. ${ }^{23,25}$

According to the literature, people with obsessive-compulsive disorders represent the group with the lowest smoking rate at $13-37 \% .^{26,27}$ Perhaps the fact that this group of patients has a strong expectation of illness, along with high levels of anxiety and perfectionist behavior, can explain the low rate. In our study, people with obsessive-compulsive disorders had SCL-90-R values $>1$ in all groups, including the control group. The highest score was found in the group with high-grade nicotine addiction $(1.51 \pm 0.83$ vs $1.21 \pm 0.63$, control group). Although these differences are not statistically significant, this issue should be researched further.

Smoking rates in psychotic disorders have been reported to be quite high at $57-66 \% .^{28,29}$ In our study, there was no correlation with the number of daily cigarettes and psychotic symptoms. However, subjects with high-grade nicotine dependence were found to have higher SCL-90-R values than the control group $(0.95 \pm 0.73$ vs $0.45 \pm 0.45$, $P=.002)$. These values are not considered significant because they are $<1$, which is the value of possible psychopathology. Our findings show that further research is needed in this regard.

There are several factors that limit our study. Because of its nature as a cross-sectional study, it was not possible to evaluate subject data after smoking cessation. In addition, the number of subjects in the mild smoker group was less than the other groups. Nevertheless, our findings, which 


\section{Psychological Features of SMOKers}

are consistent with the literature, suggest that smokers need more detailed assessment from the psychiatric side.

This study has found that, as nicotine addiction levels rise, symptoms of somatization, anxiety, depression, hostility, and paranoia gradually increase. For this reason, it is important to consider the level of nicotine addiction rather than daily cigarette consumption when evaluating psychiatric symptoms in smokers. We believe that more detailed psychiatric evaluations of these patients may be useful.

\section{Conclusion}

This study examined the association between psychological symptoms and smokers' daily cigarette consumption and nicotine addiction levels. Psychiatric symptoms (somatization, anxiety, depression, hostility, and paranoia) were observed more frequently in people with high-grade nicotine addiction (FTND score 7-10 points). The psychological symptom questionnaire may be important in identifying patients who need psychiatric counseling.

\section{REFERENCES}

1. Etter JF, Pelissolo A, Pomerleau C, De Saint-Hilaire. Associations between smoking and heritable temperament traits. Nicotine Tob Res 2003;5:401-409.

2. U.S. National Cancer Institute and World Health Organization. The economics of tobacco and tobacco control. National Cancer Institute Tobacco Control Monograph 21. 2017:7-27.

3. Abramovitch A, Abramowitz JS, Mittelman A. The neuropsychology of adult obsessive-compulsive disorder: a meta-analysis. Clin Psychol Rev 2013 Dec;33(8):1163-1171.

4. Colton CW, Manderscheid RW. Congruencies in increased mortality rates, years of potential life lost, and causes of death among public mental health clients in eight states. Prev Chronic Dis 2006 Apr; 3(2):A42.

5. Xu YM, Chen HH, Li F, Deng F, Liu XB, Yang, HC, et al. Prevalence and correlates of cigarette smoking among Chinese schizophrenia inpatients receiving antipsychotic mono-therapy. PLoS ONE 2014;9(2):e88478.

6. Kelly, C, McCreadie, R. Cigarette smoking and schizophrenia. Adv Psychiatr Treat 2000;6:327-331.

7. de Cássia Rondina, R, Gorayeb, R, Botelho, C. Psychological characteristics associated with tobacco smoking behavior. J Bras Pneumol 2007;33(5):592-601

8. American Psychiatric Association, Diagnostic and statistical manual of mental disorders (5th ed.). Washington, DC: American Psychiatric Association; 2013.

9. Shahab L, Andrew S, West R. Changes in prevalence of depression and anxiety following smoking cessation: results from an international cohort study (ATTEMPT). Psychol Med 2014 Jan;44(1):127-141.
10. Fagerström KO. Measuring degree of physical dependence to tobacco smoking with reference to individualization of treatment. Addict Behav 1978;3(3-4):235-241.

11. Derogatis LR: SCL-90-R: Administration, scoring and procedures manual for the revised version. Baltimore, MD: John Hopkins University School of Medicine; 1977

12. Dağ İ. Belirti Tarama Listesi (SCL-90-R)' nin üniversite öğrencileri için güvenilirliği ve geçerliliği. Türk Psikiyatri Dergisi 1991;2:5-12.

13. Uysal MA, Kadakal F, Karşidağ C, Bayram NG, Uysal O, Yılmaz V. Fagerstrom test for nicotine dependence: reliability in a Turkish sample and factor analysis. Tuberk Toraks 2004;52:115-121.

14. Piasecki TM, Hedeker D, Dierker LC, Mermelstein RJ. Progression of nicotine dependence, mood level, and mood variability in adolescent smokers. Psychol Addict Behav 2016;30(4):484-493.

15. McLaughlin I, Dani JA, De Biasi M. Nicotine withdrawal. Curr Top Behav Neurosci 2015;24:99-123.

16. Lawrence D, Mitrou F, Zubrick SR. Smoking and mental illness: results from population surveys in Australia and the United States. BMC Public Health 2009;9:285.

17. Tsoh JY, Humfleet GL, Muñoz RF, Reus VI, Hartz DT, Hall SM. Development of major depression after treatment for smoking cessation. Am J Psychiatry 2000;157(3):368-374.

18. Balhara YP, Jain R, Sundar SA, Sagar R. A comparative study of reliability of self report of tobacco use among patients with bipolar and somatoform disorders. J Pharmacol Pharmacother 2011;2(3):174-178.

19. Cahill K, Lancaster T, Green N. Stage-based interventions for smoking cessation. Cochrane Database Syst Rev 2010;11:CD004492.

20. West R. The multiple facets of cigarette addiction and what they mean for encouraging and helping smokers to stop. COPD 2009; 6(4):277-283

21. Becoña E, Fernández del Río E, López-Durán A, Piñeiro B, Martínez Ú. Axis II disorders and cigarette smoking among adults from the general population. J Pers Disord 2013 Jun;27(3):411-424.

22. Kutlu MG, Parikh V, Gould TJ. Nicotine addiction and psychiatric disorders. Int Rev Neurobiol 2015;124:171-208.

23. Hughes JR, Hatsukami DK, Mitchell JE, Dahlgren LA. Prevalence of smoking among psychiatric outpatients. Am J Psychiatry 1986; 143(8):993-997.

24. Byeon $\mathrm{H}$. Association among smoking, depression, and anxiety: findings from a representative sample of Korean adolescents. PeerJ 2015; 3:e1288.

25. Lasser K, Boyd JW, Woolhandler S, Himmelstein DU, McCormick D, Bor DH. Smoking and mental illness: a population-based prevalence study. JAMA 2000;284(20):2606-2610.

26. Tan $\mathrm{O}$, Taş C. Symptom dimensions, smoking and impulsiveness in obsessive-compulsive disorder. Psychiatr Danub 2015;27(4):397405

27. Abramovitch A, Pizzagalli DA, Geller DA, Reuman L, Wilhelm S. Cigarette smoking in obsessive-compulsive disorder and unaffected parents of OCD patients. Eur Psychiatry 2015;30(1):137-144.

28. Gurillo P, Jauhar S, Murray RM, MacCabe JH. Does tobacco use cause psychosis? Systematic review and meta-analysis. Lancet Psychiatry 2015;2(8):718-725.

29. Cooper J, Mancuso SG, Borland R, Slade T, Galletly C, Castle D. Tobacco smoking among people living with a psychotic illness: the second Australian Survey of Psychosis. Aust N Z J Psychiatry 2012;46(9):851-863.

This article is approved for Continuing Respiratory Care Education credit. For information and to obtain your CRCE

(free to AARC members) visit



www.rcjournal.com 